\title{
ENS-AI: Un sistema experto para la enseñanza
}

\author{
Clara BARROSO \\ Universidad de La Laguna
}

\begin{abstract}
SUMMARY.-The E.S. being developed in named ENS-AI, acronym for ENSeñanza Artificial Intilligence. ENS-AI will work as support and guide system for education practice. It is being implemented in the expert system environment MILORD-II, developed in the Institut d'Investigació en Intel.ligencia Artificial (IIIA) of the C.S.I.C. MILORD-II provides a mechanism to represent heuristic rules weighted by linguistic certainty values, based on the use of many-valued logics. This facility allows to represent poor structured knowledge domains, such as in the education case.
\end{abstract}

\section{INTRODUCCIÓN}

Es habitual en muchos grupos de trabajo pedagógico, admitir que para la actividad cognitiva del hombre es el ordenador el mejor simulador del funcionamiento de la mente. Sin entrar en tales planteamientos, de lo que no hay duda es de que el conocimiento pedagógico puede representarse como sistemas de proposiciones sobre las cuales se pueden definir operaciones computacionales: operaciones reguladas por leyes de transformación, mediante las cuales se pueden obtener inferencias. Quedará luego, el proceso de corroboración de las mismas. De igual manera, las relaciones que puedan ser definidas entre procesos de carácter pedagógico, si se conoce la naturaleza de esa relación, podrían ser representadas por funciones de diversa índole susceptible de computación.

Todo ello quiere indicar que el conocimiento humano proposicionalmente expresable, desde ese punto de vista, puede configurar un sistema experto mediante el que representar procesos educativos, relaciones entre los mismos, evaluación de estado de cosas, inferencias, toma de decisiones, etc.

Tal planteamiento constituye un campo de reflexión teórica sobre la educación de evidente interés, y perfila la utilidad de la informática como instrumento y medio para la simulación y sistematización del conocimiento utilizado de Pedagogía.

El presente trabajo, al tiempo que refleja de facto la utilidad de un sistema experto, añade una referencia sobre los procesos de su creación. Todo ello se manifiesta -lo teórico y lo práctico- mediante un ejercicio real dentro de un campo de actividad pedagógica generalizada, la característica de los equipos multiprofesionales. No se presenta el trabajo como medio para la difusión de un sistema experto que pudiera ser utilizado, sino como la demostración, como argumento, del planteamiento teórico anterior. Por lo mismo lo que se espera del lector, es la reflexión sobre el propósito y los problemas teóricos y prácticos que deja planteados. 
El estudio comienza con el planteamiento de la situación real desde la que se inicia la reflexión. A partir de ésta, se presenta la construcción del modelo para la representación del conocimiento experto en educación, y se diseña y formula el sistema experto. Este simula los procesos de reflexión propios de los expertos humanos en educación.

Los «Equipos Multiprofesionales» se ocupan del diagnóstico y la orientación educativa. Son equipos interdisciplinares compuestos por psicólogos, pedagogos y profesores de EGB, cuya labor es la de servir de apoyo y orientación al profesor, elaborando el diagnóstico psicopedagógico del alumno y orientando al profesor que se ocupa del mismo, hacia intervenciones educativas específicas para el caso que les ocupa.

Estos equipos evalúan y tratan los casos que presentan problemas dentro del ámbito escolar y son susceptibles de tratamiento en el mismo. Para llevar a cabo esta tarea, los equipos multidisciplinares demandan información sobre tres ámbitos fundamentales:

- el alumno (sus características individuales y sociales)

- su entorno de enseñanza-aprendizaje (características externas -entorno familiar y social- e internas -entorno escolar-)

- su profesor (formación, habilidades, destrezas, actitudes y expectativas)

Estos datos les permiten realizar diagnósticos educativos para recomendar estrategias de intervención educativa (tales como acciones educativas especiales o uso de metodologías didácticas alternativas) que permitan subsanar el tipo de problema diagnosticado; éstos se manifiestan habitualmente en un bajo rendimiento escolar o en conductas desadaptadas del alumno.

No obstante estos Equipos Multiprofesionales, han de hacer frente a un gran número de casos por lo que preferentemente atienden aquellos que presentan los problemas más graves, (psicopatías, casos de alumnos con necesidades educativas especiales, etc.) quedando otros casos de «menor gravedad» sin resolver (retrasos escolares, difunciones leves de conducta, etc.). El profesor ha de solventar estas situaciones anómalas sin contar, habitualmente, con equipo de apoyo alguno. En muchos de estos casos, el profesor ha de improvisar soluciones no siempre eficaces o satisfactorias.

Por un lado, es habitual que haya de abordar dichos problemas con una preparación insuficiente (la formación previa no les prepara para enfrentarse a procesos de toma de decisiones y evaluación de decisiones técnicas en casos no habituales). Por otro lado no puede percibir con suficiente objetividad los efectos de su acción docente sobre el alumno; todo ello redunda en un menor aprovechamiento de los recursos que podrían ponerse al servicio de la mejora del proceso de enseñanza-aprendizaje. Por ello parece adecuado desarrollar un instrumento que, en aquellas situaciones en que el docente no cuente con el apoyo necesario, pueda ayudarle a hacer frente a casos anómalos.

\section{EL DOMINIO DE CONOCIMIENTOS EN EDUCACIÓN}

El dominio de conocimientos en educación se caracteriza por:

- no ser «estructurado» (no existe una convención generalizada sobre los procesos de exploración y resolución de problemas)

- diferentes grados de fiabilidad en la información que utiliza (intuiciones, teorías consensuadas, conocimientos empíricos, etc.) 
- habitualmente se trabaja con informaciones incompletas o inciertas (las informaciones pueden provenir de juicios, percepciones, o datos objetivos)

- falta de consenso en las teorías básicas consideradas (distintas teorías definen métodos y estrategias alternativos en enseñanza-aprendizaje).

En consecuencia el conocimiento pedagógico empleado en la toma de decisiones prácticas, es el resultado de una compilación de conocimientos empíricos, intuiciones y teorías básicas provenientes de distintas disciplinas, y exige la utilización de estrategias de tipo heurístico para la localización y resolución de problemas.

Los expertos en educación ${ }^{1}$ usan datos conocidos para elaborar deducciones sobre los procesos de enseñanza-aprendizaje. Teniendo en cuenta que:

- las deducciones no vienen determinadas por reglas o leyes generalizables

- la construcción de hipótesis de trabajo se basa en un proceso de análisis heurístico que permite una evaluación de cada caso en concreto y en el que las intuiciones pueden ser parte del propio razonamiento

- la toma de decisiones viene siempre asociada a un determinado grado de confianza.

Los expertos en educación habitualmente razonan de lo general a lo particular. Comienzan por requerir datos básicos del caso que analizan, y realizan una primera aproximación al mismo. Usando estos datos generales construyen una primera hipótesis. Por ejemplo, si el caso que se está considerando es el de un alumno que habitualmente está distraído, que no atiende durante las explicaciones del profesor y que se muestra inquieto; se podría comenzar por considerar si dicho alumno presenta alguna disminución auditiva. Esta hipótesis sugiere la indagación sobre aspectos que, plausiblemente, estarán involucrados en este tipo de problema, lo que permitirá afirmar o desechar la hipótesis sobre la disminución auditiva. Si se desestima esta posibilidad, los expertos se dirigen a explorar otras «zonas» que pueden suministrarles informaciones más precisas o significativas para el caso que se está considerando, infiriendo un conjunto de hipótesis sobre las posibles causas del problema del alumno.

Estas hipótesis iniciales les guiarán en la exploración para acceder a informaciones más precisas que permitan contrastar nuevas posibilidades (Así, en el caso considerado, se estudiará si la causa del comportamiento anómalo del alumno puede deberse a un rechazo escolar; a una situación de retraso escolar que le esté dificultando la asimilación de los contenidos que se desarrollan en la clase; o bien si tiene algún tipo de retraso cognitivo o evolutivo que le impidan el seguimiento normal de la actividad escolar). Cada una de estas hipótesis estará asociada un determinado grado de confianza que determina en qué orden se pondrán a prueba; cuáles pueden ser desestimadas; y qué nuevas hipótesis deben ser consideradas en caso de no obtener conclusiones satisfactorias.

Este proceso de razonamiento de los expertos, persiguen descomponer en subproblemas el caso que se está considerando, a fin de realizar una búsqueda ordenada y eficaz que les permita realizar un diagnóstico sobre el problema.

1. Se ha de tener en cuenta que, en lo sucesivo, cuando nos refiramos a expertos en educación nos referiremos a profesionales (pedagogos o psicólogos no necesariamente docentes) que analizan, desde una perspectiva interdisciplinar, los fenómenos educativos. 


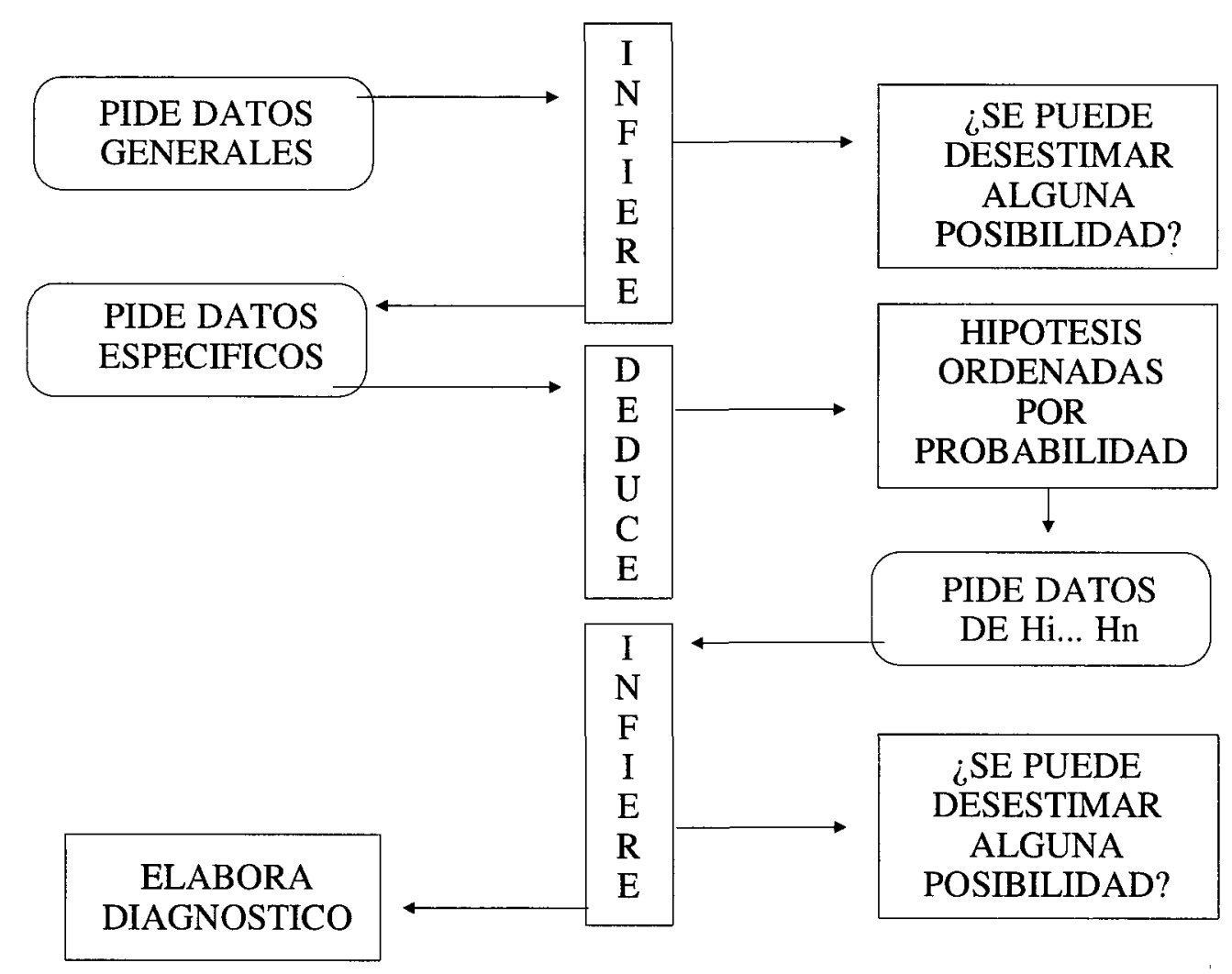

Figura 1. Tareas del experto

Dependiendo de las causas que, previsiblemente, han motivado el problema detectado, los expertos orientarán al profesor hacia determinadas soluciones metodológicas. Así el diagnóstico psicopedagógico del alumno, les permite conocer si es un problema de retraso escolar, de retraso cognitivo, si es un problema físico o de adaptación social del alumno. En este caso se recomiendan acciones educativas especiales.

El estudio de las destrezas y habilidades técnicas del profesor permite saber si el problema reside en una práctica de enseñanza inadecuada en cuyo caso se orientará al profesor en el estudio y desarrollo de metodología didáctica alternativa.

El estudio de las características del entorno de enseñanza-aprendizaje persigue detectar si éstas pueden estar influyendo en el rendimiento anómalo de alumno o del profesor para proponer acciones educativas específicas si se han de paliar carencias de este entorno.

\section{DESCRIPCIÓN DEL SISTEMA ENS-AI}

La denominación ENS-AI corresponde al acrónimo de ENSeñanza-Artificial Inteligence. En un SE capaz de simular razonamientos de expertos en educación. El objetivo final es orientar al profesor en las decisiones metodológicas más eficaces para su práctica educativa teniendo en cuenta los diagnósticos elaborados sobre las características de 
los alumnos del entorno de enseñanza-aprendizaje y las habilidades y destrezas del profesor.

El modelo conceptual de ENS-AI consta de tres grandes conjuntos de datos: alumno, profesor, entorno de enseñanza-aprendizaje. Estos, a su vez, están divididos en subconjuntos más específicos. Representan espacios de datos que, desde el punto de vista del conocimiento de los expertos en educación, permiten clasificar los datos en espacios deductivos significativos. Este modelo conceptual ha servido para decidir qué datos son más generales y constituyen la base de todo razonamiento; qué datos son específicos y sólo se han de considerar en unos determinados casos; cuándo se debe profundizar en aspectos concretos y qué hechos se pueden inferir de los datos considerados. Esta organización conceptual constituye un modelo de la $\mathrm{BC}$, que será útil para la posterior modelización con el lenguaje MILORD-II.

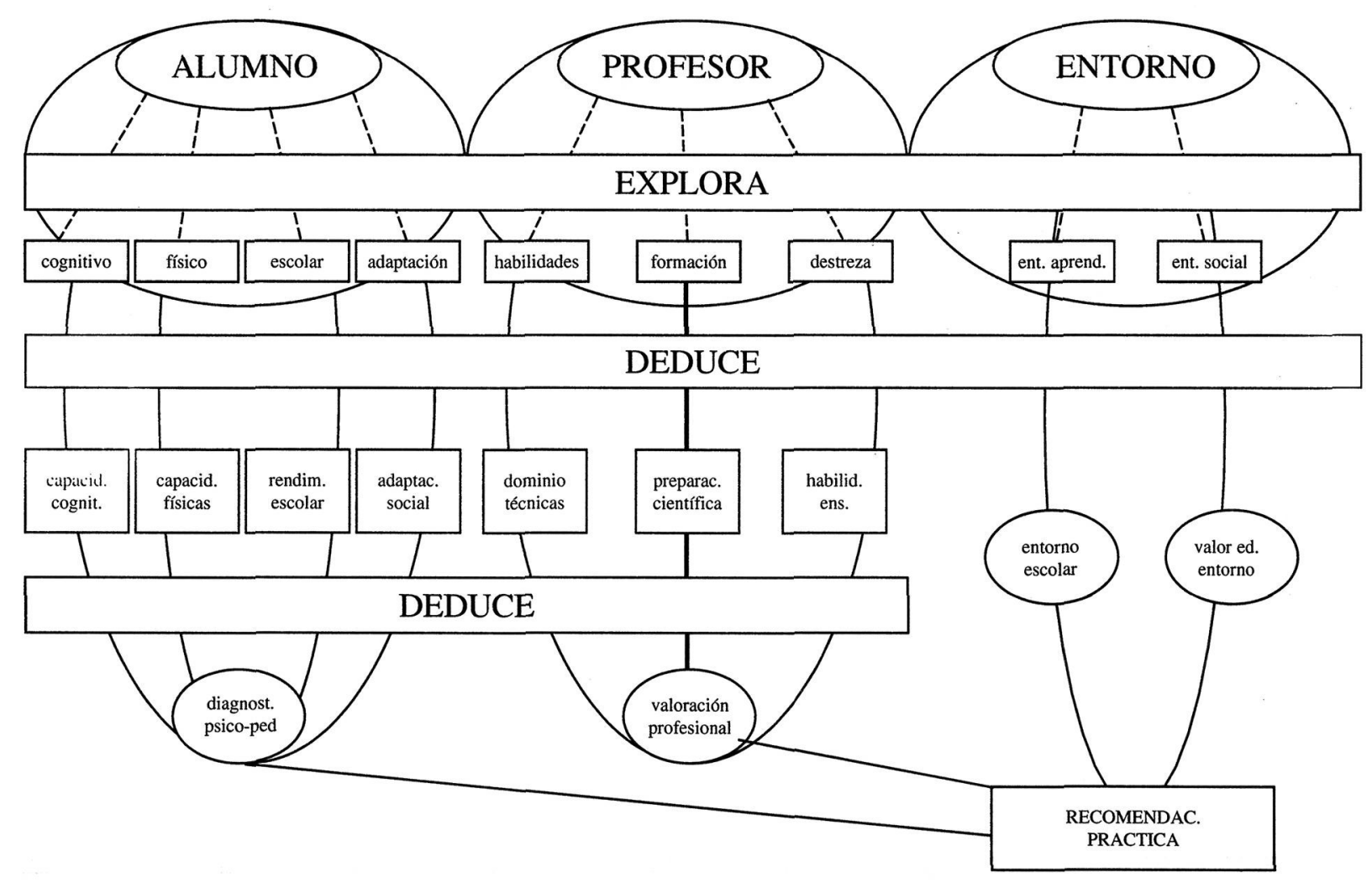

Figura 2. Modelo conceptual.

Los conjuntos de datos presentan una relación jerárquica que expresa niveles de generalidad. Existen conjuntos más generales que corresponden a los datos básicos de cada ámbito (alumno, profesor, entorno) y representan la primera aproximación que realiza el experto humano al estudiar cada ámbito. Los subconjuntos corresponden a una exploración «en profundidad» de determinados aspectos; con ellos se persigue atrapar características más precisas, que pueden tener relevancia para la decisión final sobre las recomendaciones prácticas.

Por ejemplo, el conjunto ALUMNO agrupa los datos del alumno en distintos ámbitos (cognitivo, físico, escolar y adaptación social); el subconjunto escolar informa sobre aspectos generales del rendimiento escolar del alumno, tales como su nivel en lectura, en 
cálculo, en escritura. En el subconjunto de rendimiento escolar se profundiza en el estudio del rendimiento en cada una de estas grandes áreas de conocimiento para saber, con más precisión, cual es el logro y las limitaciones del alumno en cada área concreta. Esto permite obtener informaciones precisas sobre el nivel escolar alcanzado y las destrezas que, asociadas a los objetivos cognitivos y objetivos de aprendizaje ha desarrollado el alumno.

El modelo conceptual de la BC (figura 2) es diferente del modelo del SE. Las principales diferencias entre el modelo conceptual y el modelo del SE, se deben a la necesidad de explicitar mecanismos que puedan representar el proceso de adopción y refutación de hipótesis referido anteriormente. El experto en educación utiliza estas estrategias implícitamente, desarrolla sus razonamientos de forma intuitiva; al diseñar el SE ha de declarar requerimientos de control, para así representar su proceso de indagación heurística y de razonamiento.

En síntesis, el modelo conceptual del Sistema representa la organización y clasificación de informaciones que los expertos humanos utilizan en el planteamiento, análisis y resolución de problemas. El modelo de implementación del SE, incorpora la explicitación de las heurísticas de procesamiento de información, en forma de requerimientos de control en el funcionamiento del Sistema ${ }^{2}$.

No obstante, el modelo conceptual de la BC y la implementación de la BC en el SE, comparten la organización de los hechos en conjuntos y subconjuntos (a partir de ahora denominados módulos y submódulos) así como la relación jerárquica entre módulos definida en la BC.

\section{IMPLEMENTACIÓN}

La modelización del conocimiento experto en educación ha de representar las características del dominio de conocimiento, y la incorporación de procesos deductivos y de inferencia que simulen el uso del conocimiento propio del experto humano (Derry et al., 1987; Johnson, 1988; Lawrence, 1988). Por ello se ha estimado oportuno utilizar en ENS-AI un instrumento que permite capturar los requerimientos y el modo de representar y operar con el conocimiento propio de los expertos en educación.

ENS-AI se ha implementado utilizando el entorno para SE MILORD II, desarrollado en el Institut d'Investigació en Intel-ligència Artificial (IIIA) del CSIC. Es un instrumento capaz de capturar las características de dominios de conocimiento poco estructurados, como es el caso del conocimiento en educación. El uso del entorno MILORD II permite aproximarse eficazmente al tipo de razonamientos «inciertos» o «incompletos» que involucra el conocimiento pedagógico ya que introduce, utilizando lógica multivaluada, el razonamiento aproximado como elemento que influye en una parte importante de la arquitectura del Sistema (Agustí et al., 1992; Sierra \& Godo, 1992). La principal característica de éste es la de ser modular, lo que permite:

2. En lo sucesivo, el concepto de modelo que se utiliza, queda definido como representación formal del conocimiento. En consecuencia las referencias a la representación del conocimiento, han de entenderse como la formalización del mismo. 
- utilizar la organización en módulos de la BC

- facilitar la definición de grandes aplicaciones permitiendo el diseño por partes de la BC (lo que simplifica la tarea de definición de las reglas de inferencia, siendo fácilmente utilizable por el experto)

- permite un diseño incremental de la BC y la reutilización de fragmentos de la misma que se puedan necesitar durante la ejecución del SE

- separar los elementos del dominio de conocimientos, de los elementos de control del sistema (en cada módulo).

\subsection{Implementación del dominio}

Para representar los espacios deductivos que definen conjuntos de datos en el modelo conceptual de ENS-AI (figura 2), se definen módulos y submódulos.

Un módulo se declara, en el entorno MILORD II, básicamente asignándole un identificador, una interfase de exportación y otra de exportación. También puede contener elementos de control.

El identificador de un módulo es un símbolo mediante el que se identifica al mismo en el sistema. La interfase de importación está constituida por un conjunto de hechos propios del módulo que pueden ser preguntados al usuario. La interfase de exportación es el conjunto de hechos visibles de un módulo, pueden ser informados al usuario y/o utilizados por otros módulos que accedan a éste. El módulo que pone a disposición hechos de su exportación, se convierte en submódulo de los que puedan acceder a él. Si un módulo desea disponer de datos de la interfase de exportación de otro, ha de declarar que comienza por importar los hechos que necesita del otro módulo (ej. Begin Module i = Introducción) debiendo declarar en sus reglas el identificador del módulo consultado (ej. i/etapa significa que el dato etapa pertenece al módulo Introducción y se está usando en la regla de otro módulo).

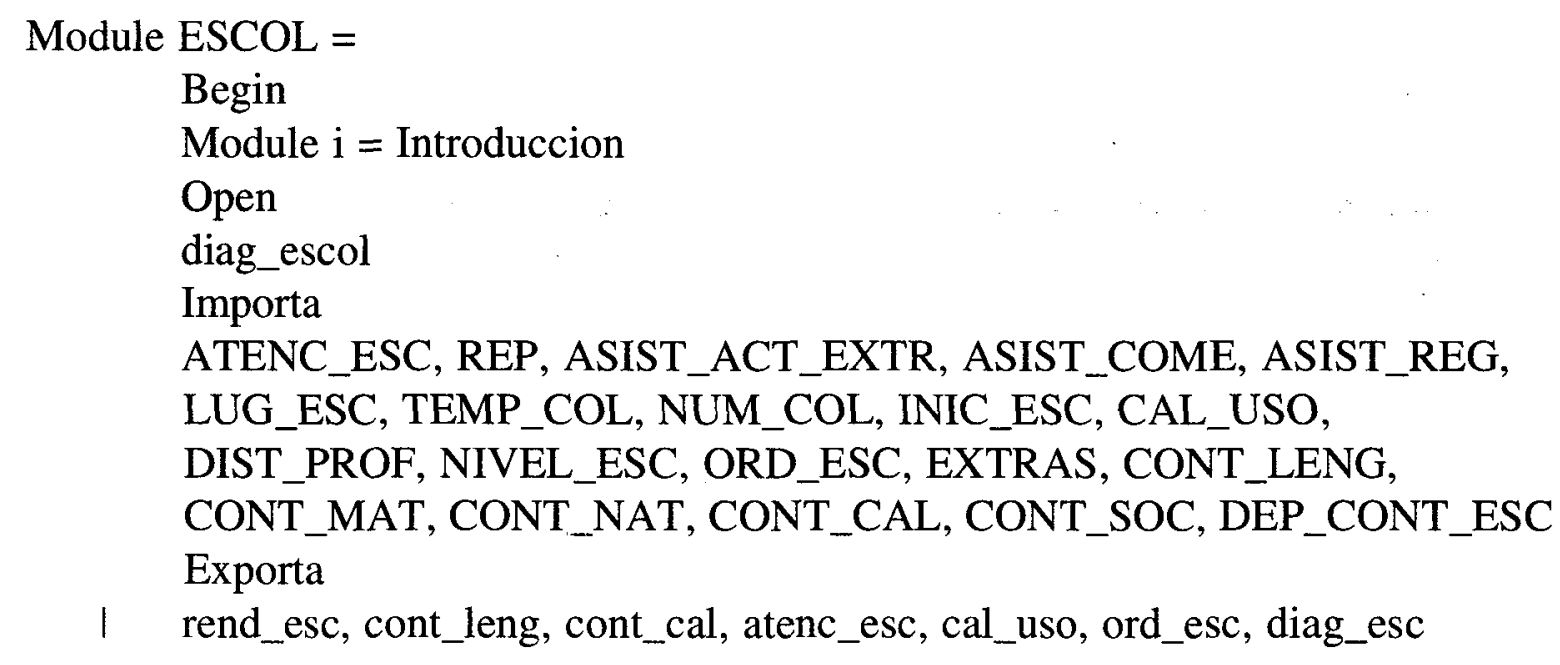

Al principio de los módulos más generales se declara con qué módulos están relacionados y qué tipo de relación existe entre ellos (ej. Module ESCOL Open diag_escol significa que el módulo diag_escol es submódulo de ESCOL es decir que este último puede adoptar decisiones sobre él para filtrarlo). Con ello se pretende modelizar los procesos 
de indagación que usa el experto razonando desde lo general a lo particular y decidiendo si ha de profundizar en el estudio de determinados aspectos o abandonarlos.

El Conocimiento Deductivo de un módulo es fundamentalmente la declaración de la $\mathrm{BC}$ que pertenece a dicho módulo. Al Conocimiento Deductivo pertenecen el Diccionario y los Predicados en los que se especifican los hechos, la definición de los hechos y las Reglas.

Conocimiento Deductivo

Diccionario:

Predicados:

Los hechos se definen en MILORD II mediante un identificador y varios atributos, de los cuales destacamos: nombre, pregunta, relacción y tipo.

Los hechos se expresan:

CONT_CAL = Nombre: $\ll$ Rendimiento especifico en calculo»

Pregunta: «Tiene especial dificultad en realizar las tareas de calculo?»

Tipo: (SI OR NON OR A_VECES)

Relaccion: needs_false cont_mat

El identificador es un símbolo mediante el que se identifica un dato o hecho en las reglas que lo usan (ej. CONT_CAL). El atributo Nombre es el nombre extenso del hecho que se declara, sirve de orientación para el experto en la elaboración de la base de conocimientos (ej. «Rendimiento específico en cálculo»). El atributo Pregunta es el que define los hechos de la interfase de exportación, la pregunta que el sistema hace al usuario (ej. «Tiene especial dificultad en resolver las tareas de cálculo?»). El atributo Relacción define la relacción entre varios hechos, declara la prioridad o subordinación de uno respecto a otro u otros decidiendo qué hechos han de ingresarse en primer lugar y, dependiendo de la respuesta a éstos, se ordena el requerimiento de datos al usuario para evitar la demanda de datos no relevantes al caso que se ejecuta e inhibe las preguntas que resulten ilógicas (ej. Relación: needs_false cont_mat significa que para preguntar CONT_CAL el hecho cont_mat ha de preguntarse antes y obtener como respuesta que es falso). El atributo Tipo es el que define el carácter del hecho.

Los hechos utilizados por ENS-AI son de distintos tipos dependiendo de los valores que puedan adoptar: booleanos, numéricos, enumerados y lógicos.

Los hechos booleanos sólo pueden tener dos valores: si o no (ej. «Escribe correctamente su nombre y apellidos?»); los hechos numéricos son aquellos cuyo valor es un número real (ej. «Qué edad tiene el alumno?»). Ambos tipos de hechos representan datos cuantitativos.

Los hechos enumerados pueden adoptar distintos valores previamente declarados, el usuario ha de elegir uno de estos valores para responder a la pregunta (ej. «Tiene especial dificultad en resolver tareas del area de lenguaje?»: SI o NO o A_VECES). Representan datos de tipo cualitativo.

Los hechos lógicos son aquellos que pueden adoptar como valor un grado de certeza (ej. rendimiento escolar es ligeramente probable). Representan los hechos deducidos en las reglas, cuyos valores posibles son: seguro, muy posible, posible, probable, ligeramente probable e imposible. 


\section{REPRESENTACIÓN DE LA BASE DE CONOCIMIENTOS}

Para representar el modelo heurístico que usa el experto en educación agrupando datos y realizando inferencias, ENS-AI utiliza reglas que relacionan datos y deducen hechos nuevos. Ej. si el alumno está en segundo curso de secundaria obligatoria y tiene dificultades en resolver las tareas de cálculo y no tiene dificultades en las tareas de lenguaje, podemos concluir que presentará un rendimiento escolar ligeramente aceptable.

En ENS-AI el valor asociado a la conclusión de la regla denota el valor de certeza del hecho deducido, éste se propaga a aquellas reglas o metarreglas que, posteriormente, pudieran utilizarlo.

Las reglas de inferencia se definen mediante un identificador (ej. R0120), un antecedente (ej. If $\mathrm{i} / \mathrm{etapa}=\left(\mathrm{S} \_2\right)$ and cont_cal $=(\mathrm{si})$ and cont_leng $\left.=(\mathrm{no})\right)$ y un consecuente (ej. rend_esc is LP).

Las reglas se expresan:

$\mathrm{R} 0120$ If $\mathrm{i} / \mathrm{etapa}=\left(\mathrm{S} \_2\right)$ and cont_cal $=(\mathrm{si})$ and cont_leng $=(\mathrm{no})$ then conclude rend_esc is LLP

La clasificación heurística de los datos; así como la construcción y contrastación de hipótesis que realizan el experto en educación, determinan la organización en módulos de la BC; predominando los criterios del dominio de conocimiento, sobre los criterios de tratamiento y control informático de los datos.

Cada módulo, en la BC, usa unos datos determinados y deduce un hecho abstracto o conceptual que no es directamente representable pero que puede inferirse a partir de éstos (En lo sucesivo se utilizará datos para designar las informaciones que provienen de la interfase de usuario; los hechos serán los datos una vez utilizados por el SE o bien los que sean deducidos por éste. Así del valor de los datos «edad» y «memoria remota», se puede inferir el valor del hecho «capacidad en memoria»). Los hechos que concluyen los módulos consultados son utilizados para elaborar un diagnóstico educativo del problema de que se trate, ponderando la imprecisión de la información obtenida, así como la incerteza del conocimiento almacenado y el conocimiento deducido.

ENS-AI trata dos clases de datos: cuantitativos y cualitativos. Los datos cuantitativos se caracterizan por representar hechos objetivos, mientras que los datos cualitativos expresan datos asociados a juicios de valor.

Datos cuantitativos:

ETAPA = Nombre: $«$ Etapa que cursa»

Pregunta: «En que etapa esta el alumno?»

Tipo: (P_1 o P_2 o P_3 o S_1 o S_2)

Datos cualitativos:

CONT_LENG = Nombre: «Rendimiento especifico en Lenguaje»

Pregunta: «Tiene especial dificultad en resolver tareas del area de lenguaje?»

Tipo: (SI o NO o A_VECES)

Los hechos concluidos por las reglas son cualitativos:

REND_ESC $=$ Nombre: «Rendimiento escolar»

Tipo: lógico 


\title{
5.1. Implementación del control
}

Como se ha apuntado en secciones anteriores, los expertos inician la exploración requiriendo datos generales, posteriormente otros más específicos; éstos les llevan a decidir qué dinámica de exploración heurística van a ir desarrollando, y qué nuevos datos han de requerir. En consecuencia, los mecanismos para establecer la exploración no son decididos arbitrariamente o a priori. Para modelizar la forma en que el experto va recorriendo los distintos espacios de datos y simular la búsqueda heurística que realiza, se utilizan varias estrategias en el Control del Sistema por medio de metarreglas que se definen en el nivel de control de cada módulo.

Las metarreglas permiten declarar las relaciones entre módulos, el orden de ejecución de los mismos, la significación de determinados datos para la activación/inhibición de uno o varios módulos y la activación/inhibición de determinadas reglas. En consecuencia, el comportamiento del sistema dependerá de la ejecución (datos ingresados y hechos deducidos) que decidirá la dirección de exploración entre módulos.

Las metarreglas se definen mediante:un identificador (ej. M0101), un antecedente (ej. IfK send-esc, (S or MP) y un consecuente (ej. then filter diag-escol).

Las metarreglas que actúan sobre los submódulos se declaran en el control estructural del módulo a que pertenecen dichos submódulos y determinan (dependiendo de los datos ingresados) la ejecución o abandono de los mismos.

\author{
Control del Sistema \\ Control Estructural \\ M0101 If $K$ (rend_esc, (S or MP)) \\ then filter \\ diag_escol
}

La modelización de la búsqueda de datos y exploración que realizaría el experto humano dentro de un módulo, está representada por las metarreglas que actúan sobre el propio módulo y definen la deducción interna que se realiza en éste. Se declara en el nivel de control de deducción de cada módulo y persigue que la exploración se efectúe eficazmente (ej. obviando el requerimiento de datos que pudieran inferirse de otros ya tratados).

Control del Sistema

Control Deductivo

M0100 If $K$ (=(e/cont_leng, (si)), S)

then conclude

$\mathrm{K}$ (cal_trans, GP)

Tanto el nivel del control deductivo como el de control del sistema, determinan qué sentido se da a la dinámica de exploración en ENS-AI, para modelizar el procedimiento del experto durante el proceso de indagación.

En el caso del ejemplo representado, los módulos Introducción y Usuario requieren del usuario datos necesarios para explorar el módulo Escol. Este permite ingresar datos generales sobre el rendimiento y nivel escolar de un alumno. A partir de los datos introducidos y de una primera deducción realizada en el módulo ESCOL, se puede decidir el nivel de rendimiento escolar del alumno con una cierta seguridad, que viene definida por 
el grado de certeza asociada al hecho deducido. Si el grado de certeza es menor de un determinado límite establecido, se pasará a explorar el módulo DIAG_ESCOL. Para ello, al principio del módulo más general se declara con qué módulos está relacionado y qué tipo de relación existe entre ellos (ej. Module ESCOL Open diag_escol significa que el módulo diag_escol es submódulo de ESCOL es decir que este último adopta decisiones sobre él para visitarlo o filtrarlo). Con ello se pretende modelizar los procesos de indagación que usa el experto razonando desde lo general a lo particular.



Figura 3. Ejemplo de exploración.

El módulo DIAG_ESCOL permite explorar con más precisión el rendimiento escolar del alumno, requiriendo datos sobre sus logros en las áreas básicas del curriculum escolar (escritura, lectura, lenguaje y cálculo). Usando los datos ingresados, se deduce el hecho: diagnóstico escolar que informa sobre qué área o áreas concretas han de ser exploradas en profundidad. Por tanto este módulo dirige hacia la exploración de los módulos CALCULO, LENGUAJE, ESCRITURA y LECTURA.

Los módulos CALCULO, LENGUAJE, ESCRITURA y LECTURA informan sobre el rendimiento específico del alumno en cada una de las áreas. A su vez, el módulo ESCRITURA decidirá sobre la exploración de ESCR_COP, ESCR_DIC y ESCR_RED; el módulo LECTURA decidirá sobre la exploración de los módulos PRE_LECT, LECT_1 y LECT_2 (que se corresponden con objetivos cognoscitivos y didácticos de distintos niveles de enseñanza) teniendo en cuenta el nivel que cursa el alumno para conocer, de forma más concreta, sus deficiencias.

Otra de las estrategias utilizadas en el control de ejecución del Sistema ENS-AI, consiste en definir módulos con distinto tipo de evaluación: eager y lazy. Esta se declara en los módulos que son responsables de dirigir la ejecución de otros más específicos. 
En los módulos cuya evaluación es de tipo eager, se efectúa una exploración en detalle de todos los hechos que exporta el módulo, así como de los hechos exportables de los submódulos que le pertenecen. En caso de obtener de esta primera exploración las informaciones necesarias para elaborar un diagnóstico con un grado de certeza aceptable, los módulos más generales desactivarán la ejecución de sus submódulos; en caso contrario, activarán la ejecución completa de uno o varios de sus submódulos a fín de obtener datos más precisos.

Los módulos cuya evaluación es de tipo lazy activarán la exploración de sus submódulos en la medida en que los datos que estos suministran sean necesarios para la consulta que se esté realizando. Al igual que los anteriores, estos módulos pueden adoptar decisiones sobre la desactivación de sus submódulos.

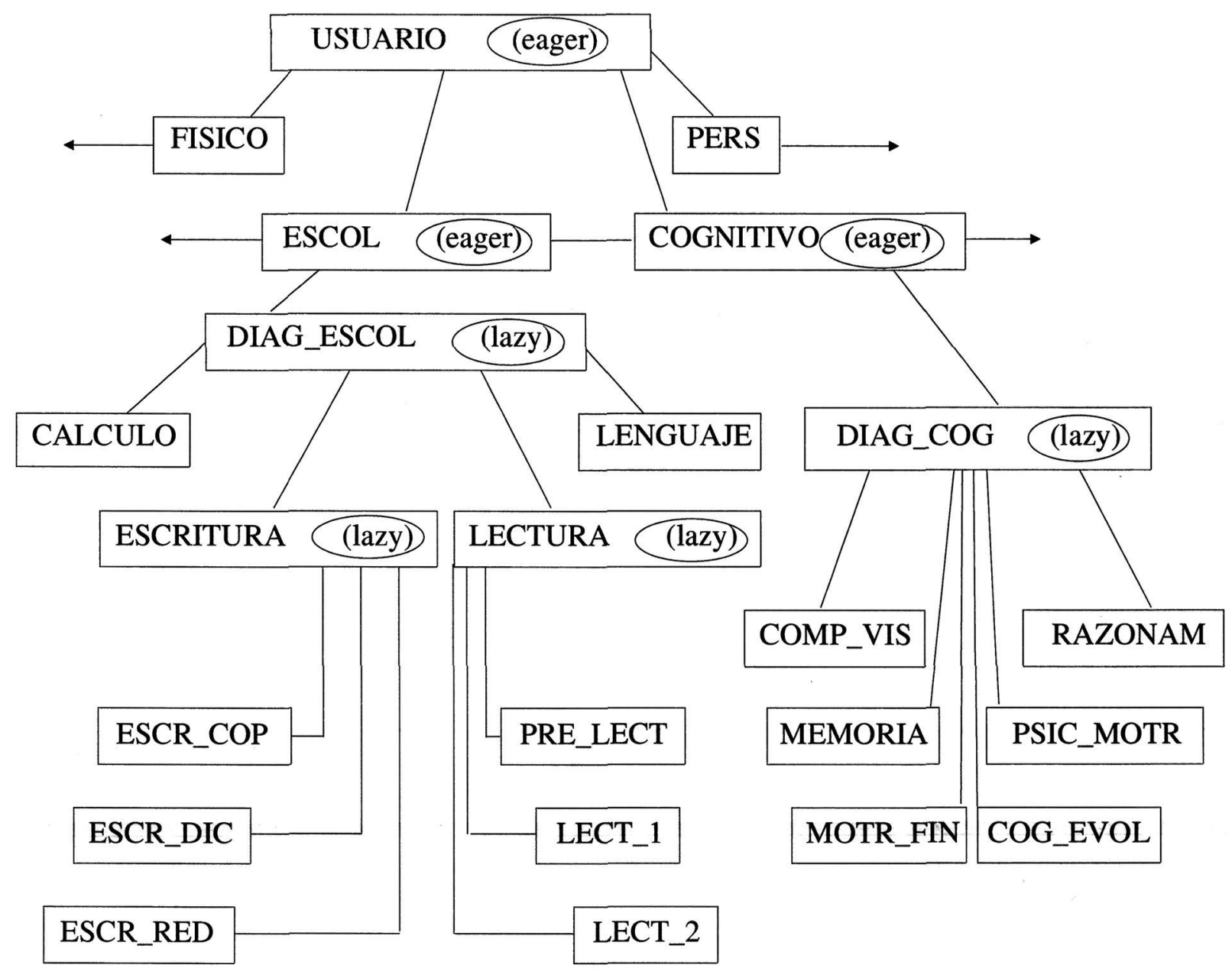

Figura 4. Ejemplo de Control.

En el ejemplo representado, el módulo COGNITIVO requiere de su submódulo DIAG_COG todos los hechos exportables. El módulo DIAG_COG sólo activará el requerimiento de datos de sus submódulos COMP_VIS, MEMORIA, MOTR_FIN, COG_EVOL, PSIC_MOTR y RAZONAMIENTO, cuando las reglas activadas durante la ejecución del Sistema así lo requiera. 
Así si el módulo COGNITIVO realiza una valoración adecuada del nivel de desarro1 lo del alumno, filtrará el módulo DIAG_COG.

Control del Sistema

Control Deductivo

M1400 If $K$ (des_al, (S o MP))

then filter

diag_cog

Si esta valoración se estima baja, se requieren por el módulo DIAG_COG los datos necesarios para explorar el nivel que tiene el alumno en comprensión visual, memoria, motricidad fina, madurez evolutiva, madurez psicomotriz y/o razonamiento.

\section{EJECUCIÓN DEL SISTEMA ENS-AI}

El profesor, usuario del sistema, introduce datos respondiendo las preguntas que le presenta ENS-AI seleccionando, de entre un conjunto de respuestas posibles, aquella que corresponde al caso. Estos datos son utilizados por la BC para inferir diagnósticos y recomendaciones de actividades educativas. Finalmente se emite una conclusión: un diagnóstico psicopedagógico del alumno, así como una valoración de las técnicas y habilidades del profesor y del entorno de enseñanza-aprendizaje, para recomendar una intervención pedagógica (asociada a un valor de certeza) que le permita enfrentarse a la solución del problema que se ha diagnosticado.

A la vez, el usuario puede obtener información adicional, a lo largo del proceso, activando la función específica de «documentación», para obtener orientaciones científicas y bibliográficas sobre el problema objeto de la consulta. Esta función informa sobre las teorías básicas que constituyen la argumentación que utiliza el razonamiento del sistema y permite el acceso a las fuentes documentales de las teorías básicas en que se basa la parte de la $\mathrm{BC}$ que ha sido activada con su consulta. Con ello se cubren dos objetivos: dar a conocer la orientación teórica de la BC (para que el usuario pueda juzgar la validez del instrumento que se le ofrece); ofrecer orientaciones de bibliografía científica actualizada. Es importante resaltar la oportunidad de esta opción ya que con ella se pretende obtener, como objetivo asociado, un instrumento que (a la vez que puede responder a las demandas de resolución de problemas concretos en actividades de enseñanza) se ofrezca como un elemento del perfeccionamiento docente.

El funcionamiento ENS-AI se realiza en un entorno de interacción con el usuario que es fácilmente asequible, de forma que el uso del sistema no requiere un costoso entrenamiento previo.

\section{CONTRIBUCIONES}

Esta investigación aporta principalmente las siguientes realizaciones:

- representa conocimiento experto en educación atendiendo a las características epistemológicas de éste

- diseña un SE dirigido al profesor como usuario

- los hechos se agrupan en módulos que permiten deducir conceptos

- la representación de la $\mathrm{BC}$ en el SE expresa heurísticas del dominio experto en educación 
- presenta una especificación de ejecución (restricciones de ejecución, control deductivo) eficaz

- el control del sistema tiene en cuenta los resultados parciales de la ejecución

- el control del sistema representa los procesos de búsqueda heurística propia del experto

- el control del sistema es declarativo.

Por último, en el desarrollo de esta aplicación, el experto en el dominio de conocimiento de la Pedagogía ha propuesto el problema a modelizar y las posibilidades que, desde su dominio percibía. Los ingenieros han establecido, desde su propio dominio, los límites dentro de los cuales era posible modelizar lo que el experto propuso. A partir de estas propuestas, el trabajo se ha desarrollado en colaboración. El experto en Pedagogía conoce la herramienta que usa para modelizar el conocimiento que posee y ha demandado instrumentos e instrucciones específicas a medida que los ha necesitado; los ingenieros han valorado si son eficaces para el problema planteado y susceptibles de realización. De esta forma se ha superado el problema, tantas veces discutido, de la primacía del experto o del ingeniero de conocimiento en el diseño y desarrollo de aplicaciones de sistemas expertos. Ha sido, por tanto, un trabajo realizado dentro de la tradición interdisciplinar de la investigación en Inteligencia Artificial.

Agradezco, en este sentido, las enseñanzas de los doctores Josep Puyol y Carlos Sierra, en el terreno de la informática aplicada (sin cuya comprensión y dedicación no habría podido abordar estos estudios); y las discusiones con el equipo de investigadores del Institut d'Investigació en Intel-ligència Artificial, que me han posibilitado el inicio de una línea nueva de estudio, y la realización del presente trabajo.

\section{BIBLIOGRAFÍA}

Agustí, J.; Esteva, F.; García, P.; Godo, L.; de Mántaras, R. L.; Murgui, L.; Puyol, J. y SIERRA, C. (1992). Structured local fuzzy logics in MILORD. En ZADEH, L. y KAPRZYK, J., editores, Fuzzy Logic for the management of Uncertainty, pp. 523-567, New York. Wiley and Sons.

Belmonte, M. A. (1990). Renoir: un sistema experto para la ayuda en el diagnóstico de colagenosis y artropatías inflamatorias. Tesis Doctoral, Universitat Autónoma de Barcelona.

DERRY, S. J.; HAWKES, L. y TSAI, C. (1987). A theory for remediating problem-solving skills of older children and adults. Educational Psychologist, 22:55-87.

JoHnson, E. (1988). Expertise and decision under uncertainty: Performance and process. En The Nature of Expertise. Lawrence Erlbaum Associates, Inc.

LAWRENCE, J. (1988). Expertise on the bench: Modeling magistrates' judicial decision-making. En The Nature of Expertise. Lawrence Erlbaum Associates, Inc.

PuYOL, J.; Agustí, J. y SIERRA, C. (1991). Partial evaluation in milord-ii: A languaje for knowledge engineering. En Proceedings Europ-IA'92, pp. 193-207.

PuYOL, J.; Godo, L. y SiERRA, C. (1992). A specialisation calculus to improve expert systems communication. En Proceedings Europ-IA'92, pp. 144-148.

PUYOL, J. (1994). Modularization, Uncertainty, Reflective Control and Deduction by Specilization in MILORD II, a Language for Knowledge-Based Systems. Tesis Doctoral, Universitat Autónoma de Barcelona. 
SIERRA, C. y Godo, L. (1992). Specifying simple scheduling task in a reflective and modular arquitecture. En Proceedings of ECAI'92, pp. 177-206.

VERDAGUER, A. (1989). Pneumon-IA: desenvolupament $i$ validació d'un sistema expert d'ajuda al diagnostic medic. Tesis Doctoral, Universitat Autónoma de Barcelona. 